\title{
Analytic Approach to the MSSM Mass Spectrum in the Large Tan $\beta$ Regime.
}

\author{
E. G. Floratos ${ }^{(*)}$ \\ Institute of Nuclear Physics, \\ NSRC Demokritos, \\ Athens, Greece \\ and \\ G. K. Leontaris ${ }^{(* *)}$ \\ Centre de Physique Theorique, Ecole Polytechnique, \\ F-91128 Palaiseau, France
}

\begin{abstract}
In various unified extensions of the Minimal Supersymmetric Standard Model, the Yukawa couplings of the third generation are predicted to be of the same order. As a result, low energy measured mass ratios, require large ratios of the standard model Higgs vacuum expectation values, corresponding to a large value of the parameter $\tan \beta$. We present analytic solutions for the Yukawa couplings and the Higgs and third generation squark masses, in the case of large top and bottom Yukawa couplings. We examine regions of these Yukawas which give predictions for the top mass compatible with the present experimentally determined top mass and provide useful approximate formulae for the scalars. We discuss the implications on the Radiative Symmetry Breaking mechanism and derive constraints on the undetermined initial conditions of the scalars.
\end{abstract}

IOA $320 / 95$

hep-ph/9503455

${ }^{(*)}$ On leave of absence from Phys. Dept., University of Crete, Iraklion,Crete, Greece

${ }^{(* *)}$ On leave of absence from Phys. Dept., University of Ioannina, 45110 Ioannina, Greece 


\section{Introduction}

If one adopts the idea of unification of all forces, then the most natural candidate which is at the same time the simplest extension of the standard model (SM) of strong and electroweak interactions, is the minimal supersymmetric standard model (MSSM). Indeed, the low energy measured values of the three gauge couplings are consistent with simple unification values at an energy of $\mathcal{O}\left(10^{16} \mathrm{GeV}\right)$, provided that the SM - spectrum is extended to that of the MSSM at an energy scale $M_{S U S Y}^{e f f} \sim 1 T e V$. Furthermore, unified supersymmetric models solve successfully the hierarchy problem[1] of their corresponding non-supersymmetric versions. Recently, in view of the possible observation of supersymmetric particles in the upcoming colliders, there has been a growing interest in the determination of the complete mass spectrum of MSSM. Nevertheless, although the MSSM is considered as a successful extension of the SM, it still has a number of arbitrary parameters which are not fixed by the theory. It is expected however that these parameters could be reduced by embedding the MSSM in a more fundamental theory such as supergravity or superstrings. In the present status of MSSM and its Grand Unified extensions, (SUSY-GUTs), the Yukawa couplings and the initial conditions for the scalar mass terms are treated as arbitrary parameters of the theory. Most of the SUSY-GUTs predict certain relations between the Yukawa couplings of the third generation, up to an overall constant which is left arbitrary. Certain string constructions may relate this constant to the unified value of the common gauge coupling at the string scale, usually up to a factor of $\mathcal{O}(1)$. Most common relations that hold in these models 2] are

$$
h_{t} \sim h_{b} \sim h_{\tau}, h_{t}>h_{b} \sim h_{\tau} \text { or } h_{t} \sim h_{b}>h_{\tau}
$$

Remarkably, it was shown by numerical analyses [3], that the top mass is predicted to be $m_{t} \sim 180 \mathrm{GeV}$ which is compatible with the recent experimental findings[ [⿴囗十. Furthermore, in the context of MSSM, the top mass has been calculated in the recent literature mainly by numerical methods or semi-analytically in the case of the exact equality $h_{t}=h_{b}$ [5, 6]. In the above cases, the experimentally observed low energy hierarchy $m_{t} \gg m_{b}$ demands a large ratio of the two Higgs vacuum expectation values (vevs), $\tan \beta \equiv \frac{v_{2}}{v_{1}} \gg 1$.

As far as the scalar masses are concerned, the aforementioned theories have not been very predictive yet. In the simplest treatments, one usually assumes that all scalar masses (i.e. higgses and soft susy masses ) have the same boundary condition at the unification scale (universality), while their splittings at low energies 
arise mainly from the renormalisation group running. In general, however, one expects that supergravity or superstrings will induce non-universal conditions for the scalar masses.

In a certain class of supergravity models [7], each scalar mass $\tilde{m}_{i}$ can be parametrised in terms of a coefficient $\xi_{i} \sim \mathcal{O}(1)$ (calculable in specific models), and the supersymmetry breaking scale which is proportional to the gravitino mass $m_{3 / 2}$ corresponding to an approximately flat direction of the underlying fundamental theory. In the effective low energy theory, the over all scale - set by the gravitino mass $m_{3 / 2}$ - is not known, since $m_{3 / 2}$ depends on singlet scalar field vevs of the higher unknown theory. It has been claimed however, that since $m_{3 / 2}$ is a field dependent quantity, its value can be determined dynamically by minimizing [8] the vacuum energy of the effective low energy theory with respect to $m_{3 / 2}$. This procedure has shown that under general assumptions for the higher theory and a wide choice of $\xi_{i}$ 's 9 , the $m_{3 / 2}$ scale is of the required order, i.e. $\sim \mathcal{O}\left(m_{Z}\right)$. This tells us that supersymmetry is broken at the right scale to protect scalars from large radiative corrections, while the resulting susy scalar spectrum is accessible by the future colliders.

While non-universality is not an inescapable prediction of the fundamental theory, it is a necessity for large $\tan \beta$. Indeed, if we are to take seriously predictions of exact or approximate $h_{t}, h_{b}$ equality at $M_{U}$, the radiative breaking of the $S U(2) \times U(1)$ gauge symmetry [10] cannot be realized naturally if we assume universal boundary conditions - in particular for the higgs mass parameters.

In the present work, we wish to explore the region of the parameter space of $\xi_{i}=\tilde{m}_{i}^{2} / m_{3 / 2}^{2}, \ldots$ which naturally leads to radiative symmetry breaking of the electroweak gauge group, protecting simultaneously the scalar quarks from receiving negative masses. This latter phenomenon is quite possible due to large negative radiative corrections on the scalars of the third generation. We should also point out that another possible consequence of using non-universal boundary conditions for scalars 11] is the appearance of enhanced flavor changing neutral currents (FCNCs) 12]. To suppress FCNCs within the experimentally accepted limits, it is adequate to consider only cases diagonal in the flavor space. In our analysis we are going to derive semi-analytic formulae which will prove useful as will be explained in the next section.

Our paper is organized as follows: In section 2, in order for the paper to be self contained, we start with a short review of the low energy effective supersymmetric theory and explain our notation. We proceed by presenting the analytic solution for large $h_{t}-h_{b}$ couplings and comment on their applicability to the minimization 
conditions of the low energy potential with respect to higgs vevs and the gravitino mass. In section 3 we apply our solutions to derive analytic expressions for various useful quantities (including $\tan \beta$ ). Section 4 deals with the coupled differential equations for scalar mass of the third generation in the regime of large $\tan \beta$. It is shown that the solution of the system of equations for all third generation scalar masses is reduced to one simple first order equation of Riccati type. We derive analytic solutions in the limiting cases of low and large $\tan \beta$ cases and explore the non-universal conditions which are compatible with RSB. Finally, in section 5 we present our numerical results and conclusions. 


\section{Large Top-Bottom Couplings in MSSM.}

The theory which concerns us here is a supersymmetric low energy effective theory based on the general structure of spontaneously broken $N=1$ supergravity [13]. Although such a theory still leaves a lot of arbitrariness, in the minimal case the effective theory can be described in terms of five new parameters only, namely the gravitino mass term $m_{3 / 2}$ the $m_{1 / 2}, \mu, m_{3}^{2}=B \mu$ mass parameters and the trilinear parameter $A$. In the present paper however, for reasons explained already in the introduction, we will assume non-universality for the higgs and the other scalar mass parameters at the unification scale $M_{U}$, thus our parameter space will be enlarged. At the tree level, the masses of the fermions are obtained from the trilinear superpotential given by

$$
\mathcal{W}=h_{Q}^{i j} Q_{i} u_{j}^{c} H_{2}+h_{D}^{i j} Q_{i} d_{j}^{c} H_{1}+h_{L}^{i j} L_{i} e_{j}^{c} H_{1}+\mu H_{1} H_{2}
$$

Supersymmetry is broken softly through the "soft - breaking " terms which provide with masses all the s-particles. Thus, the gauginos $\lambda_{i}$ receive masses from terms $\frac{1}{2} \tilde{m}_{i} \lambda_{i} \bar{\lambda}_{i}$. Scalar mass terms arise from the effective potential which in the minimal case is of the form

$$
\mathcal{L}=\Sigma\left|\frac{\partial \mathcal{W}}{\partial \phi_{i}}\right|^{2}+\bar{A} m_{3 / 2}\left(\mathcal{W}+\mathcal{W}^{*}\right)+m_{3 / 2}^{2} \Sigma\left|\phi_{i}\right|^{2}+\bar{B} m_{3 / 2}\left(\Sigma \phi_{i} \frac{\partial \mathcal{W}}{\partial \phi_{i}}+\text { h.c. }\right)+\ldots
$$

where the $\{\ldots\}$ represent $D$-term contributions. $\bar{A}, \bar{B}$ are parameters of $\mathcal{O}(1)$ and in the case of non-universality they may have different values for each superpotential term, i.e. $\bar{A}=\left\{\bar{A}_{Q}, \bar{A}_{D}, \ldots\right\}$ etc. Thus, generalizing the Lagrangian in (8) for the non-universal case, one obtains soft squark and slepton mass terms of the form $\tilde{m}_{n}^{2} \tilde{q}_{n}^{*} \tilde{q}_{n}$, where $q_{n}=\left\{Q_{n}, u_{n}^{c}, d_{n}^{c}, L_{n}, e_{n}^{c}, \nu_{n}\right\}$. Moreover, due to the trilinear superpotential couplings in (2) there correspond soft-susy breaking trilinear couplings of the form

$$
A_{Q} h_{Q}^{i j} Q_{i} u_{j}^{c} H_{2}+A_{D} h_{D}^{i j} Q_{i} d_{j}^{c} H_{1}+A_{L} h_{L}^{i j} L_{i} e_{j}^{c} H_{1}+B \mu H_{1} H_{2}
$$

where now $A_{n}=\left(\bar{A}_{n}+3 \bar{B}_{n}\right) m_{3 / 2}$, with $n=Q, D, \ldots$ and $B=\left(\bar{A}_{\mu}+2 \bar{B}_{\mu}\right) m_{3 / 2}$.

The RG-improved effective potential for the neutral particles can be written as follows

$$
\begin{aligned}
V_{0}(Q) & =\left(m_{H_{1}}^{2}+\mu^{2}\right)\left|H_{1}\right|^{2}+\left(m_{H_{2}}^{2}+\mu^{2}\right)\left|H_{2}\right|^{2}+m_{3}^{2}\left(H_{1} H_{2}+\text { h.c. }\right) \\
& +\frac{g^{2}+g^{\prime^{2}}}{8}\left(\left|H_{1}\right|^{2}-\left|H_{2}\right|^{2}\right)^{2}+\Delta V_{1}-\eta(Q) m_{3 / 2}^{4}
\end{aligned}
$$


where 14

$$
\Delta V_{1}=\frac{1}{64 \pi^{2}} \operatorname{Str} \mathcal{M}^{4} \ln \left(\frac{\mathcal{M}^{2}}{Q^{2}}-\frac{3}{2}\right)
$$

is the one loop contribution, necessary to stabilize the minimization with respect to the higgs vevs $v_{i}=<H_{i}>$, while

$$
\operatorname{Str}_{\mathcal{M}}^{2}(z, \bar{z})=\sum_{n}(-1)^{2 s_{n}}\left(2 s_{n}+1\right) m_{n}^{2}(z, \bar{z})
$$

is the sum over all particles with spin $s_{n}$ and mass $m_{n}$.

The term $\eta(Q) m_{3 / 2}^{4}$ comes from the underlying supergravity theory and can be interpreted [8] as a contribution to the cosmological constant. Notice that terms of $\mathcal{O}\left(m_{3 / 2}^{2} M_{P l}^{2}\right)$ are not included since such contributions would destroy the hierarchy $m_{3 / 2} \ll M_{P l}$. Specific examples with these desirable features may be found in four dimensional string models [15].

The quantum corrections to the above potential depend on the particle spectrum masses which are computed with the use of R.G.E.s in terms of the initial values at the unification scale. A precise knowledge of the R.G. effects in the low energy theory is important not only from the phenomenological point of view (measurements of $\sin ^{2} \theta_{W}, \alpha_{e m}$, top mass, e.t.c.) but for theoretical reasons as well. In particular the Higgs mass parameters and squark masses of the third generation play a very important role in the radiative breaking of the electroweak symmetry on the one hand and the preservation of the $S U(3)$ confining group and the electric charge on the other.

From phenomenological analyses, two limiting cases have been of particular interest. The first, is when the two higgs vev's are of the same order $(\tan \beta \sim$ $\mathcal{O}(1))$ while the difference between the top mass and the other fermion masses is attributed to a large top Yukawa coupling relative to the bottom, $h_{t} \gg h_{b}$.

It is interesting that this case can easily be treated analytically both in the fermion and scalar mass sector at the one loop level [16, 17]. Since $h_{t}$ is the only non-negligible Yukawa, all the others can be ignored and the relevant R.G.E.s decouple, leading to first order differential equations which can be solved by standard methods. The same procedure can be used for the scalars including those of the third generation.

The case with a large bottom Yukawa coupling $(\tan \beta \gg 1)$ is much more complicated. In a previous work [18], however, it was shown that if one neglects the small differences in the $U(1)_{Y}$ factors, an analytic solution of the $h_{t}-h_{b}$ coupled differential system can be obtained easily. In particular, in the absence 
of a large tau Yukawa coupling, one can write the $h_{t}-h_{b}$ equations at the 1-loop as follows

$$
\begin{aligned}
\frac{d}{d t} h_{t}^{2} & =\frac{1}{8 \pi^{2}}\left\{6 h_{t}^{2}+h_{b}^{2}-G_{Q}\right\} h_{t}^{2} \\
\frac{d}{d t} h_{b}^{2} & =\frac{1}{8 \pi^{2}}\left\{h_{t}^{2}+6 h_{b}^{2}-G_{B}\right\} h_{b}^{2}
\end{aligned}
$$

with

$$
G_{Q}=\sum_{i=1}^{3} c_{Q}^{i} g_{i}^{2}, \quad G_{B}=\sum_{i=1}^{3} c_{B}^{i} g_{i}^{2}
$$

where $c_{Q}^{i}=\left\{\frac{16}{3}, 3, \frac{13}{15}\right\}$ and $c_{B}^{i}=\left\{\frac{16}{3}, 3, \frac{7}{15}\right\}$ for $S U(3), S U(2)$ and $U(1)$ respectively.

In order to solve Eqs(8-9), we define the new parameters $x, y$ through the relations $h_{t}^{2}=\gamma_{Q}^{2} x, h_{b}^{2}=\gamma_{Q}^{2} y$, with

$$
\begin{aligned}
\gamma_{Q}^{2} & =\exp \left\{\frac{1}{8 \pi^{2}} \int_{t}^{t_{0}} G_{Q}\left(t^{\prime}\right) d t^{\prime}\right\} \\
& =\prod_{j=1}^{3}\left(1-\frac{b_{j, 0} \alpha_{j, 0}}{2 \pi}\left(t-t_{0}\right)\right)^{\frac{c_{Q}^{j}}{b_{j}}}
\end{aligned}
$$

and make the following transformation

$$
u=\frac{k_{0}}{(x-y)^{5 / 6}} \equiv \frac{k_{0}}{\omega^{5 / 6}}, \quad d I=\frac{6}{8 \pi^{2}} \gamma_{Q}^{2} d t
$$

where the parameter $k_{0}=4 x_{0} y_{0} /\left(x_{0}-y_{0}\right)^{7 / 6}$ depends on the initial conditions $x_{0} \equiv h_{t, 0}^{2}$ and $y_{0} \equiv h_{b, 0}^{2}$. Then, we can form a differential equation for the new variable $u$ which can be cast in the form [18

$$
\frac{u^{1 / 5} d u}{\sqrt{1+u}}=-\frac{5}{6} k_{0}^{6 / 5} d I
$$

Then, by simple integration it can be shown that the solution can be given in terms of hypergeometric functions, ${ }_{2} F_{1}(a, b, c ; z)$, i.e.

$$
u^{7 / 10}{ }_{2} F_{1}\left(\frac{1}{2}, \frac{-7}{10}, \frac{3}{10}, \frac{1}{-u},\right)-u_{0}^{7 / 10}{ }_{2} F_{1}\left(\frac{1}{2}, \frac{-7}{10}, \frac{3}{10}, \frac{1}{-u_{0}},\right)=\frac{7}{12} k_{0}^{6 / 5} I(t)
$$

with $I(t)=\frac{3}{4 \pi^{2}} \int_{t_{0}}^{t} \gamma_{Q}^{2}\left(t^{\prime}\right) d t^{\prime}$. Thus, for a given set $x_{0}, y_{0}$ of initial conditions at $M_{U}$ we can calculate the $u, \omega(t)$ values from equation (14) and determine the $h_{t}, h_{b}$ from

$$
h_{t}^{2} \equiv \gamma_{Q}^{2} x
$$




$$
\begin{aligned}
& =\gamma_{Q}^{2} \frac{1}{2} \omega\left(1+\sqrt{1+k_{0} \omega^{-5 / 6}}\right) \\
h_{b}^{2} & \equiv \gamma_{Q}^{2} y \\
& =\gamma_{Q}^{2} \frac{1}{2} \omega\left(-1+\sqrt{1+k_{0} \omega^{-5 / 6}}\right)
\end{aligned}
$$

In ref [6] similar solutions where proposed. A further study of the solutions by these authors showed that all $h_{t, b_{0}}>1$ initial values accumulate on a curve on the $h_{t}, h_{b}$ plane. In the region $h_{t} \approx h_{b}$, this curve is slightly deformed when the difference of the hypercharge in the two $\mathrm{RG}$-equations is taken into account. $\mathrm{A}$ numerical fit has shown the relation [6]

$$
\left(h_{t}-0.015\right)^{12}+\left(h_{b}^{2}+0.045\right)^{12}=\left(\frac{\gamma_{Q}}{\sqrt{I / 2}}\right)^{12}
$$

The main deformation occurs around the diagonal, i.e. when $h_{t} \sim h_{b}$. The top mass solutions presented in the subsequent section are slightly affected by this change. In any case, this uncertainty on the top mass is in fact overwhelmed by much bigger uncertainties arising from sparticle loop corrections on the bottom mass. A more detailed discussion of the latter will be presented in the next section. For most applications, (in particular when $u \gg 1$ ), it is appropriate to expand the hypergeometric function and obtain a simplified form of the above solutions. They can be expressed in terms of a simplified form of the function $\omega(t)$

$$
\omega(t)=\frac{x_{0}-y_{0}}{\left\{{ }_{2} F_{1}^{0}+\frac{7}{6} \sqrt{x_{0} y_{0}} I(t)\right\}^{12 / 7}}
$$

where the value of the hypergeometric function at $u=u_{0}$ is ${ }_{2} F_{1}^{0} \approx 1$ for the limit of interest, i.e. for $h_{t, 0} \sim h_{b, 0}$. Formula (18) is going to be used later in the calculations for the scalar masses. Note finally (for later use) the useful relation between $x, y$ variables

$$
\left(\frac{x-y}{x_{0}-y_{0}}\right)^{7}=\left(\frac{x y}{x_{0} y_{0}}\right)^{6}
$$

In particular, in certain supergravity theories the Yukawas are subject to various constraints which may be combined with the above formulae and result to interesting predictions [8, 19].

The analytic $h_{t}-h_{b}$ solution neglects small differences arising from the different hypercharge assignment of the top and bottom quarks. This approximation is quite reasonable since other uncertainties (neglect of Yukawa couplings, one loop approximation e.t.c) of the same order are also introduced in the analytic solution. In any case, it is clear that the vastly different values of $m_{t}$ and $m_{b}$ 
masses cannot be attributed to effects due to these small corrections unless a considerable fine tuning of the parameters occurs.

The usefulness of the analytic expressions for $h_{t}-h_{b}$ couplings in the large $\tan \beta$ regime, is largely exhibited in the case of the scalar mass calculations. As has already been mentioned, the knowledge of the scalar mass parameters affected by the large Yukawa couplings at low energies, is very crucial and decisive for the particular supergravity or string model under investigation [20]. To be more concrete, let us assume that only $h_{t}, h_{b}$ Yukawas are large. In this case the up and down squark masses of the third generation as well as the two higgs mass parameters of the standard model $\left(m_{H_{i}}^{2}, i=1,2\right)$ doublets receive large negative contributions proportional to integrals involving $h_{t}^{2}, h_{b}^{2}$. If $h_{b} \ll h_{t}$, and $h_{t}$ is large enough, then $m_{H_{2}}^{2}$ mass parameter turns to a negative value at low energies triggering the $S U(2)_{L} \otimes U(1)_{Y}$ breaking radiatively. On the other hand, $m_{H_{1}}^{2}$ mass does not receive large negative corrections since $h_{b}$ coupling is small. This is of course desirable since (as it turns out in this case), the neutral Higgs potential is stable and the vev's of the Higgses are finite as expected. Besides it can be proved [17] that for the experimentally accepted top quark mass and in the largest part of the $\left(m_{1 / 2}, m_{3 / 2}\right)$ parameter space the squared masses of the top-squarks receiving negative contributions are not driven negative, protecting in this way the color and charge quantum numbers.

The situation is much more complicated in the case of large bottom coupling too. Then the other Higgs mass $m_{H_{1}}^{2}$ may also be driven negative at low energies. In order to make the analysis more transparent, let us write the conditions obtained from minimization of the Higgs potential, with respect to $v_{i}$ 's

$$
\begin{aligned}
m_{3}^{2} & =-\frac{1}{2}\left(m_{H_{1}}^{2}+m_{H_{2}}^{2}+2 \mu^{2}\right) \sin 2 \beta \\
m_{Z}^{2} & =2 \frac{m_{H_{1}}^{2}-m_{H_{2}}^{2} \tan ^{2} \beta}{\tan ^{2} \beta-1}-2 \mu^{2}
\end{aligned}
$$

In the large $\tan \beta$ scenario that we are interested here $(\tan \beta \gg 1)$ we may approximate the above formulae so that $m_{Z}^{2} \simeq-2\left(m_{H_{2}}^{2}+\mu^{2}\right)$ and $-m_{3}^{2} \tan \beta \simeq m_{H_{1}}^{2}+$ $m_{H_{2}}^{2}+2 \mu^{2}>0$. Combined together, these equations lead to $m_{H_{1}}^{2}-m_{H_{2}}^{2}>m_{Z}^{2}$ and $\mu_{1}^{2} \equiv \mu^{2}+m_{H_{1}}^{2}>\frac{m_{Z}^{2}}{2}>0$ with the conclusion that the stability of the potential is ensured only for a positive value of $\mu_{1}^{2}$ mass. Another compelling reason is also the protection of the color and charge quantum numbers. The above description shows clearly the necessity of obtaining analytic solutions of the scalar mass parameters in the case that $\tan \beta \gg 1$.

A final comment we would like to make concerning the usefulness of the pro- 
posed semi - analytic procedure is also based on theoretical motivations. Indeed, as we have already mentioned, a particularly interesting idea is that the vacuum energy density could be minimized with respect to the gravitino mass $m_{3 / 2}$ in order to fix the mass of the latter - and therefore the supersymmetry breaking scale - dynamically. The minimization of the vacuum energy with respect to the $m_{3 / 2}$ parameter leads to a certain condition for the renormalised scalar mass parameters and Higgs mass terms at low energies, which reads [8]

$$
2 V_{1}(Q)+\eta(Q) m_{3 / 2}^{4}=0
$$

while the coefficient $\eta(Q)$ obeys a renormalisation group equation of the form

$$
\frac{d \eta(Q)}{d \log (Q)}=-\frac{1}{128 \pi^{2}} \operatorname{Str} \mathcal{M}^{4}
$$

The dynamical determination of the $m_{3 / 2}$ scale requires the knowledge of the evolved soft (mass) ${ }^{2}$-parameters involved in the above constraint. Thus, analytic knowledge of their dependence on the boundary conditions are very useful. In particular, in the case of non - universal boundary conditions of the scalars at $M_{U}$, the parameter space at $M_{U}$ can be represented by a vector $\vec{\xi}=\left(\xi_{H_{1}}, \xi_{H_{2}}, \xi_{Q}, \ldots\right)$ where $\tilde{m}_{i}^{2}\left(M_{U}\right)=\xi_{i} m_{3 / 2}^{2}$. It is not easy to handle numerically the above system in this case and therefore an approximate analytic solution of the scalar mass parameters would be very important.

In what follows we wish to extend our previous results of the $t-b$ analytic expressions. We give expressions for various useful quantities including $\tan \beta$ and derive simplified formulae for $h_{t, b}$ Yukawa couplings. In particular, in the next section we develop the formalism which is going to be used in section 4 for the derivation of the scalar mass analytic expressions. 


\section{Phenomenological analysis of $b-t$ solutions}

Let us define two new parameters $\sigma$ and $\tau$

$$
\begin{aligned}
\tau & =\exp \left\{\frac{3}{4 \pi^{2}} \int_{t_{0}}^{t} h_{t}^{2} d t^{\prime}\right\} \\
\sigma & =\exp \left\{\frac{3}{4 \pi^{2}} \int_{t_{0}}^{t} h_{b}^{2} d t^{\prime}\right\}
\end{aligned}
$$

The $h_{t}-h_{b}$ RGE's can be combined to give

$$
\frac{\dot{\tau}}{\dot{\sigma}}=\frac{x_{0}}{y_{0}}\left(\frac{\tau}{\sigma}\right)^{\frac{11}{6}}
$$

where dots stand for derivatives with respect to $t=\ln (Q)$. Integration of the above gives

$$
\frac{1}{x_{0}} \frac{1}{\tau^{5 / 6}}-\frac{1}{y_{0}} \frac{1}{\sigma^{5 / 6}}=\frac{1}{x_{0}}-\frac{1}{y_{0}}
$$

Rewriting now $h_{t}-h_{b}$ RGE's in terms of $(\sigma, \tau)$ and substituting into (27) we obtain

$$
\begin{aligned}
\tau \sigma & =\frac{x-y}{x_{0}-y_{0}} \\
& =\left(\frac{u_{0}}{u}\right)^{\frac{6}{5}}
\end{aligned}
$$

where $u_{0} \equiv u\left(x_{0}, y_{0}\right)$. On the other hand, we can express the ratio $\frac{\tau}{\sigma}$ in terms of the integral

$$
\begin{aligned}
\frac{\tau}{\sigma} & =\exp \left\{\frac{3}{4 \pi^{2}} \int_{t_{0}}^{t}\left(h_{t}^{2}-h_{b}^{2}\right) d t\right\} \\
& \equiv \exp \left\{\int_{t_{0}}^{t}(x-y) d I\right\}
\end{aligned}
$$

which, with the help of (13, 12), can be expressed as follows

$$
\frac{\tau}{\sigma}=\left(\frac{y_{0}}{x_{0}} \frac{\sqrt{1+u}+1}{\sqrt{1+u}-1}\right)^{\frac{6}{5}}
$$

The explicit dependence of $\sigma, \tau$ parameters on the new variable $u$ defined in (12) is not only necessary for the scalar mass solutions we are going to discuss in the next section; it will also prove useful in other low energy parameters. Let us for example consider the evolution of the ratio of the two higgs vev's $(\tan \beta)$, in the case we are examining here, i.e. when only $h_{t, b}$ couplings are large. In this case we have the following evolution equation

$$
\frac{d}{d t} \tan \beta=-\frac{1}{4} \frac{3}{4 \pi^{2}}\left(h_{t}^{2}-h_{b}^{2}\right) \tan \beta
$$


Integration of the above gives

$$
\begin{aligned}
\tan \beta & =\tan \beta_{0}\left(\frac{\sigma}{\tau}\right)^{\frac{1}{4}} \\
& =\tan \beta_{0}\left(\frac{h_{t, 0}}{h_{b, 0}}\right)^{\frac{3}{5}}\left(\frac{\sqrt{1+u}-1}{\sqrt{1+u}+1}\right)^{\frac{3}{10}}
\end{aligned}
$$

Thus $\tan \beta$ evolution is also determined solely from the parameter $u$ and the initial values of the two Yukawas. From (32) it is obvious that for large $u$ values the $u$-dependent term in (32) tends to unity and the ratio $R_{\beta}=\tan \beta / \tan \beta_{0}$ is given approximately by $\left(h_{t, 0} / h_{b, 0}\right)^{3 / 5}$.

In the most general case, it would be interesting to combine the above result with the $\left(m_{t}, m_{b}\right)$ mass relation to eliminate the parameter $u$. Notice that to obtain this relation in the case of $\tan \beta \gg 1$ one should take into account corrections to the bottom mass, arising from loop-graphs[21, 22, 23] containing supersymmetric particles. It is possible however to assume the existence of PQ- or R-type symmetries which may prevent the appearance of such large corrections 22]. For the sake of simplicity we will assume that due to some kind of symmetry such corrections are absent. In this case one finds

$$
m_{t}=m_{b} \frac{h_{t, 0}}{h_{b, 0}} \tan \beta_{0}\left(\frac{\tan \beta_{0}}{\tan \beta}\right)^{\frac{2}{3}}
$$

where $m_{b}$ is the running bottom mass at the scale $Q=m_{t}$, related to the bottom mass at $Q \sim m_{b}$ with a renormalisation group factor $\eta_{b} \approx 1.41$, i.e., $m_{b}\left(m_{b}\right)=$ $\eta_{b} m_{b}\left(m_{t}\right)$. In figure 1 we show the variation of $r_{\beta} \equiv\left(\tan \beta / \tan \beta_{0}\right)^{2 / 3}$ vs the parameter $u$ whose range $\left(u_{0}, u_{f}\right)$ is determined from the initial values of the couplings $h_{t, 0}, h_{b, 0}$ and the solution (14). ( $u_{f}$ corresponds to the value that $u$ receives at the scale $m_{t}$ ). Since we are interested for large $\tan \beta$ values, we plot $r_{\beta}$ for several $h_{t, 0} / h_{b, 0}$ - ratios close to unity. In all cases we observe a small change of $r_{\beta}$ which approaches asymptotically its maximal value, $\left(h_{t, 0} / h_{b, 0}\right)^{\frac{2}{5}}$, as $u \rightarrow \infty$. Therefore, in these cases putting all together, one finds the simple relation

$$
m_{t} \approx m_{b}\left(m_{t}\right) \tan \beta
$$

where $m_{b}\left(m_{t}\right)$ is the $m_{b}$ mass at the scale $Q=m_{t}$.

In Table I, we present selected values of the top mass, in the case where the top Yukawa coupling is close to its non-perturbative value, $\left(h_{t, 0}=3.0\right)$ and various values of the $t / b$ Yukawa ratio close to unity, $h_{t, 0} / h_{b, 0} \geq 1$, i.e. when $h_{b, 0}$ Yukawa coupling is also large. Both, running and physical top masses are shown while in 
the last column we present the corresponding values of $\tan \beta$, assuming a central value for the bottom quark, i.e. $m_{b}\left(m_{b}\right)=n_{b} \frac{v}{\sqrt{2}} h_{b} \cos \beta=4.25 \mathrm{GeV}$. Here, as already explained, $n_{b}$ includes the running from the scale $Q \sim m_{t}$ down to the scale $m_{b}$ and is taken to be $n_{b} \approx 1.41$. We start the running at the unification scale $M_{U}$, using as inputs the unification scale itself and the value of the common gauge coupling $\alpha_{U}$ at $M_{U}$ together with the initial values of the Yukawa couplings $h_{t, 0}, h_{b, 0}$ as shown in the Table I. Then, in obtaining the low energy values of $\alpha_{e m}$, $a_{3}$, and $\sin ^{2} \theta_{W}$, we use the following ranges

$$
\alpha_{e m}{ }^{-1}=127.9 \pm .1, a_{3}=.12 \pm .01, \sin ^{2} \theta_{W}=.2319 \pm 0.0004
$$

For the large $h_{t, 0}$ values chosen here, the top mass is in remarkable agreement with the recent experimental data [4].

Of course the accuracy of the results of the table should not be overestimated. A more precise calculation should involve uncertainties arising from the strong coupling measurements and other parameters as the bottom corrections mentioned earlier. Our aim here is to show that our solutions result to stable $m_{t}, m_{t}^{p h}, \tan \beta$ predictions against $h_{t, 0} / h_{b, 0}$ variations.

\begin{tabular}{ccccc}
\hline$h_{t, 0}$ & $h_{t, 0} / h_{b, 0}$ & $m_{t} / \mathrm{GeV}$ & $m_{t}^{p h} / \mathrm{GeV}$ & $\tan \beta$ \\
\hline 3.0 & 1.17 & 176.7 & 184.7 & 58.22 \\
& 1.25 & 177.0 & 184.9 & 58.10 \\
& 1.30 & 177.1 & 185.0 & 58.06 \\
& 1.36 & 177.2 & 185.2 & 57.90 \\
& 1.42 & 177.3 & 185.3 & 57.78
\end{tabular}

Table I. $m_{t}$ and $\tan \beta$ values for $h_{t, 0}=3.0$ and various $h_{t, 0} / h_{b, 0}-$ ratios shown in the second column. We fix $m_{b}=n_{b} \frac{v}{\sqrt{2}} h_{b} \cos \beta=4.25 \mathrm{GeV}$. Large sparticle loop corrections on the bottom mass may change the above predictions.

Indeed, as has been already noticed, uncertainties from sparticle loops contributing to $m_{b}$ are not included. Therefore, the analytic solutions of the scalar masses (presented in the subsequent section) which are involved in the relevant loops may considerably simplify the examination of the above corrections. The $\delta m_{b}$ corrections will have a direct impact on the determination of the angle $\beta$ and therefore on the top-mass. The relevant loop-graphs 21] lead to the following result 22, 23]

$$
\delta\left(m_{b}\right)=\mu \frac{\tan \beta}{16 \pi^{2}}\left(\frac{8 g_{3}^{2}}{3} m_{\tilde{g}} I\left(m_{\tilde{b}, 1}^{2}, m_{\tilde{b}, 2}^{2}, m_{\tilde{g}}^{2}\right)+h_{t}^{2} A_{t} I\left(m_{\tilde{t}, 1}^{2}, m_{\tilde{t}, 2}^{2}, \mu^{2}\right)\right),
$$


where the function $I(x, y, z)$ is given by

$$
I(x, y, z)=\frac{x y \ln (x / y)+y z \ln (y / z)+x z \ln (z / x)}{(x-y)(y-z)(x-z)},
$$

In the above, $m_{\tilde{g}}$ is the gaugino mass, while $m_{\tilde{b}, i}, m_{\tilde{t}, i}$ are the eigenmasses of sbottom and stop. A crucial role is played by the relative sign of the two terms involved in the above formula. The gaugino mass is given by its one loop formula, $m_{\tilde{g}}=\frac{a_{3}}{a_{G}} m_{1 / 2}$. Our analytic procedure in the next section can also provide a simple formula for the trilinear coupling $A_{t}=f_{t}\left(A_{t, 0}, A_{b, 0}\right)-I_{\tau}^{\prime} m_{1 / 2}$, where $f_{t}$ is a linear function of $A^{\prime}$ 's while $I_{\tau}^{\prime}$ an integral to be discussed in the subsequent section. For reasonable $A_{(t, b), 0}$ initial values $A_{t}$ can be approximated $A_{t} \approx-I_{\tau}^{\prime} m_{1 / 2}$. Thus, there is a relation between the trilinear parameter and the gaugino mass, $A_{t} \approx-0.6 m_{\tilde{g}}$ which can simplify our discussion. Therefore, there is a partial cancelation of the above two terms in the formula, but in the case $\tan \beta \approx(50-60)$ as the table shows, the correction is still large.

A precise estimation depends on the details of the scalar mass spectrum involved in the integral functions $I(x, y, z)$ and the specific initial conditions of the scalars. This is nevertheless possible for any set of initial conditions on the scalars with the help of the analytic solutions of the next section. Furthermore the sign of $\mu$ plays also a decisive role. It can be easily seen that such corrections can lie in a wide range, from a few up to $40 \%$. Evidently, similar graphs to those considered above, can also result to corrections on the tau mass. Nevertheless, in this case it is found 23] that they are at most $5 \%$ and do not affect essentially the top mass prediction. It is worth noting here that in particular unified models as in the case of $S U(5) \times U(1)$ 27, the $b-\tau$ Yukawa couplings are not necessarily equal at the Unification scale. Thus, if these corrections come with the right sign, they may reconcile large top mass values, with the condition $h_{b}>h_{\tau}$. In such cases, our present analytic procedure where the $h_{\tau}$ coupling is ignored in valid within all the parameter space. 


\section{Analytic Solutions for the $3^{r d}$ Generation Scalar Masses}

We have already pointed out that in unified supersymmetric models where only $h_{t}, h_{b}$ couplings are large, the differential equations determining the evolution of the soft scalar masses of the third generation and the two Higgs mass parameters $m_{H_{1,2}}$ are coupled. In the general case with non-zero trilinear parameters $A_{t}, A_{b}$, these equations take the form 20

$$
\begin{gathered}
\frac{d \tilde{m}_{Q_{L}}^{2}}{d t}=-\frac{1}{8 \pi^{2}} \sum c_{i}^{Q} M_{i}^{2} g_{i}^{2}+\frac{h_{t}^{2}}{8 \pi^{2}} \mathcal{M}_{U}^{2}+\frac{h_{b}^{2}}{8 \pi^{2}} \mathcal{M}_{D}^{2}+\frac{1}{6} \frac{\alpha_{1}}{2 \pi} S \\
\frac{d \tilde{m}_{U}^{2}}{d t}=-\frac{1}{8 \pi^{2}} \sum c_{i}^{U} M_{i}^{2} g_{i}^{2}+2 \frac{h_{t}^{2}}{8 \pi^{2}} \mathcal{M}_{U}^{2}-\frac{2}{3} \frac{\alpha_{1}}{2 \pi} S \\
\frac{d m_{H_{2}}^{2}}{d t}=-\frac{1}{8 \pi^{2}} \sum c_{i}^{H} M_{i}^{2} g_{i}^{2}+3 \frac{h_{t}^{2}}{8 \pi^{2}} \mathcal{M}_{U}^{2}+\frac{1}{2} \frac{\alpha_{1}}{2 \pi} S \\
\frac{d \tilde{m}_{D}^{2}}{d t}=-\frac{1}{8 \pi^{2}} \sum c_{i}^{D} M_{i}^{2} g_{i}^{2}+2 \frac{h_{b}^{2}}{8 \pi^{2}} \mathcal{M}_{D}^{2}+\frac{1}{3} \frac{\alpha_{1}}{2 \pi} S \\
\frac{d m_{H_{1}}^{2}}{d t}=-\frac{1}{8 \pi^{2}} \sum c_{i}^{H} M_{i}^{2} g_{i}^{2}+3 \frac{h_{b}^{2}}{8 \pi^{2}} \mathcal{M}_{D}^{2}-\frac{1}{2} \frac{\alpha_{1}}{2 \pi} S
\end{gathered}
$$

where

$$
\begin{aligned}
& \mathcal{M}_{U}^{2} \equiv \tilde{m}_{Q_{L}}^{2}+\tilde{m}_{U}^{2}+m_{H_{2}}^{2}+A_{t}^{2} \\
& \mathcal{M}_{D}^{2} \equiv \tilde{m}_{Q_{L}}^{2}+\tilde{m}_{D}^{2}+m_{H_{1}}^{2}+A_{b}^{2}
\end{aligned}
$$

and the initial condition for $S$, in the case of MSSM, is given by

$$
S_{0}=\left(m_{H_{2}}^{2}-m_{H_{1}}^{2}\right)_{0}+\sum_{g e n}\left(\tilde{m}_{Q}^{2}+\tilde{m}_{D}^{2}+\tilde{m}_{E}^{2}-\tilde{m}_{L}^{2}-2 \tilde{m}_{U}^{2}\right)_{0}
$$

In the case of the universal boundary conditions the $S$ contribution vanishes identically as required by the absence of gravitational mixed anomaly [24]. In this case $S$ vanishes at any scale as can be deduced by integrating its corresponding renormalisation group equation, $S(t)=\frac{\alpha_{1}(t)}{\alpha_{1,0}} \operatorname{Tr}\left[Y m^{2}\right]$. It is obvious from the above equations that if we start with the same or almost equal $h_{t}, h_{b}$ couplings, the two Higgses are going to evolve in a similar way. As discussed in the case of Yukawas, again small differences of $U(1)$ factors in the scalar mass system cannot be enough to generate a difference of $m_{H_{1}}^{2}$, and $m_{H_{2}}^{2}$ running masses at $m_{W}$. Here, however, in contrast to the $h_{t}-h_{b}$ case, one has two possibilities of generating $m_{H_{1}}^{2}-m_{H_{2}}^{2}>m_{Z}^{2}$ at $m_{W}$. The first is due to the difference of 
the Yukawa couplings $h_{t, b}$. In fact the low $\tan \beta$ regime is the limiting case of this possibility. One uses for economy universal b.c.'s for scalar masses at $M_{U}$, therefore $m_{H_{1}}^{0}=m_{H_{2}}^{0}$ and the difference arising at $m_{W}$ is only due to the fact that $h_{t} \gg h_{b}$. Here, if $h_{b} \sim \mathcal{O}\left(h_{t}\right)$, universal conditions cannot possibly lead to $m_{H_{1}}^{2} \neq m_{H_{2}}^{2}$, at least not for the biggest part of the parameter space. A complete analysis of the parameter space $\left(m_{1 / 2}, m_{3 / 2}, \mu, \ldots\right)$ at $M_{U}$ is obviously required to determine those regions which are compatible with the radiative symmetry breaking and the phenomenological expectations. The isolation of the RSBcompatible regions of $\left(m_{3 / 2}, m_{1 / 2}\right)$ will have a further consequence in low energy phenomenology: it will evidently constrain the region of the supersymmetric sparticle spectrum masses. Such a constrained situation may serve as a guide in the experimental searches for their detection.

Up to know, analytic solutions exist only in the case where $h_{t} \gg h_{b, \tau, \ldots}$ [16, 17. In order to solve the above equations in the case of $h_{t} \sim h_{b}$, we form the differential equations for two sums of the above system, namely for $\mathcal{M}_{U}^{2}, \mathcal{M}_{D}^{2}$. Here we will work only in the limiting case where $A_{b, t} \rightarrow 0$. In fact, by recalling the same arguments used in the solution of scalar masses for the case $h_{t, 0} \gg h_{b, 0}$ [17] we can conclude that in the IR-limit the contributions of the trilinear parameters $A_{t}, A_{b}$ do not play important role in the final solutions for the scalar masses as long as $m_{1 / 2} \leq m_{3 / 2}$. Therefore, to simplify the subsequent analysis, we drop $A_{U}, A_{D}$ terms (the extension of the solution to the most general case is straightforward). It is easily then observed that one can write the equations for the sums of scalar masses in the following form:

$$
\begin{aligned}
\frac{d \mathcal{M}_{U}^{2}}{d t} & =\frac{1}{8 \pi^{2}}\left\{6 \mathcal{M}_{U}^{2} h_{t}^{2}+\mathcal{M}_{D}^{2} h_{b}^{2}-G_{U}^{0} m_{1 / 2}^{2}\right\} \\
\frac{d \mathcal{M}_{D}^{2}}{d t} & =\frac{1}{8 \pi^{2}}\left\{\mathcal{M}_{U}^{2} h_{t}^{2}+6 \mathcal{M}_{D}^{2} h_{b}^{2}-G_{D}^{0} m_{1 / 2}^{2}\right\}
\end{aligned}
$$

where $G_{U}^{0}=G_{Q}+G_{H_{2}}+G_{U^{c}}$ and $G_{D}^{0}=G_{Q}+G_{H_{1}}+G_{B^{c}}$. In this final system, we also observe that the $S$ contribution is no longer present in the equations. Indeed, the two sums formed above include the partners of trilinear terms of the superpotential. Due to the $U(1)$ invariance of the Yukawa Lagrangian, the $S$ term is identically zero in the sums.

To simplify the above coupled equations we make the following transformations

$$
\begin{aligned}
& \mathcal{M}_{U}^{2}=\tau \mathcal{X}, \tau=\exp \left\{\frac{3}{4 \pi^{2}} \int_{t_{0}}^{t} h_{t}^{2} d t^{\prime}\right\} \\
& \mathcal{M}_{D}^{2}=\sigma \mathcal{Y}, \sigma=\exp \left\{\frac{3}{4 \pi^{2}} \int_{t_{0}}^{t} h_{b}^{2} d t^{\prime}\right\}
\end{aligned}
$$


Then Eqs(45,46) can be written in the form

$$
\begin{aligned}
\tau \frac{d \mathcal{X}}{d t} & =\frac{1}{6} \frac{d \sigma}{d t} \mathcal{Y}-\frac{G_{U}^{0}}{8 \pi^{2}} m_{1 / 2}^{2} \\
\sigma \frac{d \mathcal{Y}}{d t} & =\frac{1}{6} \frac{d \tau}{d t} \mathcal{X}-\frac{G_{D}^{0}}{8 \pi^{2}} m_{1 / 2}^{2}
\end{aligned}
$$

Using the formulae of the previous section, the above equations can be written as follows

$$
\frac{d}{d t}\left(\begin{array}{c}
\mathcal{X} \\
\mathcal{Y}
\end{array}\right)=\mathcal{H}(t)\left(\begin{array}{c}
\mathcal{X} \\
\mathcal{Y}
\end{array}\right)-\frac{m_{1 / 2}^{2}}{8 \pi^{2}}\left(\begin{array}{c}
\frac{G_{U}^{0}}{\tau} \\
\frac{G_{D}^{0}}{\sigma}
\end{array}\right)
$$

with

$$
\mathcal{H}(t)=\gamma_{Q}^{2}(\sigma \tau)^{\frac{7}{12}}\left[\begin{array}{cc}
0 & y_{0}\left(\frac{\sigma}{\tau}\right)^{\frac{17}{12}} \\
x_{0}\left(\frac{\tau}{\sigma}\right)^{\frac{17}{12}} & 0
\end{array}\right]
$$

We may use Green's functions techniques to solve this non-homogeneous system. For this we need first the solution of the homogeneous part.

If we define the function $h(u)$ as follows

$$
h(u)=\left(\frac{x_{0}}{y_{0}}\right)^{\frac{6}{5}}\left(\frac{\sqrt{1+u}-1}{\sqrt{1+u}+1}\right)^{\frac{17}{10}}
$$

the homogeneous part becomes

$$
\begin{aligned}
& d \mathcal{X}=-\frac{1}{10} \frac{d u}{\sqrt{u^{2}+u}} h(u) \mathcal{Y}(u) \\
& d \mathcal{Y}=-\frac{1}{10} \frac{d u}{\sqrt{u^{2}+u}} \frac{1}{h(u)} \mathcal{X}(u)
\end{aligned}
$$

There is a rather complicated dependence on $u$ through $h(u)$, however, it is possible to use new parameters which may simplify the system. For example, if we define

$$
d Q=-\frac{1}{10} \frac{d u}{\sqrt{u^{2}+u}}
$$

and

$$
\frac{1}{\sqrt{1+u}}=\sin 2 \phi
$$

we can easily find that

$$
Q=\frac{1}{5} \ln (\tan \phi)
$$


Now the homogeneous system becomes

$$
\begin{aligned}
& \frac{d \mathcal{X}}{d Q}=\tilde{h}(Q) \mathcal{Y}(Q) \\
& \frac{d \mathcal{Y}}{d Q}=\frac{1}{\tilde{h}(Q)} \mathcal{X}(Q)
\end{aligned}
$$

If we seek solutions of the form

$$
\mathcal{X}=\operatorname{Exp}\left(\int \tilde{h} A d Q\right), \mathcal{Y}=\operatorname{Exp}\left(\int \frac{B}{\tilde{h}} d Q\right)
$$

then it can be shown that the unknown functions $A, B$ satisfy the relation $B=$ $1 / A$ while we find that $A$ obeys the first order Differential Equation

$$
\frac{d A}{d Q}=A\left(\frac{1}{\tilde{h} A}-\tilde{h} A\right)
$$

The latter, is a first order Riccati type Equation of the general form $\dot{A}+A^{2}-$ $f(x)=0$ whose solutions are known only for particular forms of the function $f(x)$. In our case, $x=\int \tilde{h}(Q) d Q$ and $f(x)=1 / \tilde{h}(Q)^{2}$, which is a rather complicated function of the new parameter $x$. Therefore exact analytic solutions are possible only in particular limiting cases. We can study the above equation by geometric methods and obtain useful information about its solutions. Such a mathematical analysis, however, is beyond the purposes of this paper.] We will present soon, two limiting cases which are of particular interest from the physics point of view. Before we proceed to the presentation of the interesting limiting cases let us exhibit some interesting properties of the equation (61). The above equation remains invariant when $A$ is shifted by a function $g(Q)$, i.e. $A \rightarrow A+g$, which obeys the equation

$$
\begin{aligned}
g(Q) & =\frac{g_{0} \operatorname{Exp}\left(-2 \int A \tilde{h} d Q\right) d Q}{1+g_{0} \int \tilde{h} \operatorname{Exp}\left(-2 \int A \tilde{h} d Q\right) d Q} \\
& =\frac{g_{0}}{1+g_{0} \int \tilde{h} \mathcal{X}^{-2} d Q^{\prime}} \frac{1}{\mathcal{X}^{2}}
\end{aligned}
$$

Furthermore, the equation $A(u)^{2}-h(u)^{2}=0$ defines a curve which separates the areas where the derivative of $A(u)$ changes sign. Therefore above (below) that curve, $A(u)$ is an increasing (decreasing) function of the parameter $u$. This is depicted in figure 2. Let us also discuss the possibility of searching for a simplified solution of the system (58,59). We observe from figure 2 that for approximately

\footnotetext{
${ }^{1}$ see for example [25]
} 
equal couplings, the function $h(u)$ is approximately constant within the biggest range of the parameter $u$. In figure 3 we compare the numerical solution for the homogeneous part of the differential system with the one obtained by considering $h(u) \approx$ const., assuming non-universal boundary conditions. We can conclude that the approximate analytic solution is in good agreement with the numerical one for $h_{t} / h_{b} \leq 1.1$. In what follows, we will consider approximated formulae in the interesting case of approximately equal top - bottom Yukawas. In terms of the variable $Q$ the complete system is written

$$
\frac{d}{d Q}\left(\begin{array}{c}
\mathcal{X} \\
\mathcal{Y}
\end{array}\right)=\left[\begin{array}{cc}
0 & \tilde{h}(Q) \\
\frac{1}{\tilde{h}(Q)} & 0
\end{array}\right]\left(\begin{array}{l}
\mathcal{X} \\
\mathcal{Y}
\end{array}\right)-\left(\begin{array}{c}
G_{U} \\
G_{D}
\end{array}\right)
$$

where where $G_{U, D}(Q)$ are properly defined through (50).

With the use of the diagonalizing matrix,

$$
\mathcal{U}(\tilde{h})=\frac{1}{\sqrt{2} \tilde{h}}\left[\begin{array}{cc}
\tilde{h} & -\tilde{h} \\
1 & 1
\end{array}\right]
$$

we can write the Differential Equations as follows

$$
\frac{d}{d Q}\left(\begin{array}{c}
\tilde{\mathcal{X}} \\
\tilde{\mathcal{Y}}
\end{array}\right)=\left\{\left[\begin{array}{rr}
1 & 0 \\
0 & -1
\end{array}\right]-\frac{17}{2 \sqrt{u}}\left[\begin{array}{ll}
1 & 1 \\
1 & 1
\end{array}\right]\right\}\left(\begin{array}{l}
\tilde{\mathcal{X}} \\
\tilde{\mathcal{Y}}
\end{array}\right)-\mathcal{U}^{-1}\left(\begin{array}{l}
G_{U} \\
G_{D}
\end{array}\right)
$$

(where the new vector $\Omega^{T}=(\tilde{\mathcal{X}}, \tilde{\mathcal{Y}})$ is related to the old one $\Omega_{0}^{T}=(\mathcal{X}, \mathcal{Y})$ with the matrix $\left.\mathcal{U}, \Omega=\mathcal{U}^{-1} \Omega_{0}\right)$. For the case under investigation, for example, this form is particularly useful. In the limit $h_{t, 0} \approx h_{b, 0}$, the parameter $u \gg 1$, thus the second term in the right hand side may be considered as a first order perturbation. There is another interesting limiting case which can be also easily treated, i.e., when $\sqrt{u} \ll 17$ in the range of integration. In that case (which corresponds to $\left.h_{t} \gg h_{b}\right)$, we may define the new variable $P=\ln (h)$ and the transformation

$$
\mathcal{V}=\frac{1}{\sqrt{2}}\left[\begin{array}{rr}
1 & 1 \\
1 & -1
\end{array}\right]
$$

the differential system can be written as

$$
\frac{d}{d P}\left(\begin{array}{l}
\hat{\mathcal{X}} \\
\hat{\mathcal{Y}}
\end{array}\right)=\left\{17\left[\begin{array}{ll}
1 & 0 \\
0 & 0
\end{array}\right]+\sqrt{u}\left[\begin{array}{ll}
0 & 1 \\
1 & 0
\end{array}\right]\right\}\left(\begin{array}{l}
\hat{\mathcal{X}} \\
\hat{\mathcal{Y}}
\end{array}\right)-\mathcal{V}^{-1} \mathcal{U}^{-1}\left(\begin{array}{l}
G_{U} \\
G_{D}
\end{array}\right)
$$

This particular case is treated more easily if one ignores $h_{b}$ coupling. Particular expressions are found in the literature [16, 17]. Thus, in the present work 
we restrict ourselves in the approximate bottom-top equality. It can be easily concluded in that case that $u \gg 1$ always and the function $h(u)$ is approximately constant, $h(u) \approx\left(\frac{x_{0}}{y_{0}}\right)^{6 / 5}$. In this case $\mathrm{Eq}(67)$ can be integrated, leading to the following result

$$
\left(\begin{array}{c}
\mathcal{X} \\
\mathcal{Y}
\end{array}\right)=\operatorname{Exp}\left[\mathcal{H}_{0}\left(Q-Q_{0}\right)\right]\left\{\left(\begin{array}{c}
X_{0} \\
Y_{0}
\end{array}\right)+\int_{Q_{0}}^{Q} d Q^{\prime} \operatorname{Exp}\left[-\mathcal{H}_{0}\left(Q^{\prime}-Q_{0}\right)\right]\left(\begin{array}{c}
G_{U} \\
G_{D}
\end{array}\right)\right\} .
$$

where, due to the property $\mathcal{H}^{2} \equiv\left(\begin{array}{ll}1 & 0 \\ 0 & 1\end{array}\right)$, the exponents can be expanded as follows

$$
\operatorname{Exp}\left[\mathcal{H}_{0}\left(Q-Q_{0}\right)\right]=\left(\begin{array}{cc}
\operatorname{ch}\left(Q-Q_{0}\right) & h_{0} \operatorname{sh}\left(Q-Q_{0}\right) \\
h_{0}^{(-1)} \operatorname{sh}\left(Q-Q_{0}\right) & \operatorname{ch}\left(Q-Q_{0}\right)
\end{array}\right)
$$

In the large $\tan \beta$ regime and taking into account the $Q(u)$ dependence of $\operatorname{Eq}(55)$, for the present purposes the above solutions can be approximated as follows

$$
\begin{aligned}
& \frac{\mathcal{M}_{U}^{2}}{m_{0}^{2}} \approx \frac{\tau}{2}\left\{\left(\xi_{U}+h_{0} \xi_{D}\right) \rho+\left(\xi_{U}-h_{0} \xi_{D}\right) \frac{1}{\rho}\right\}+\xi_{1 / 2}<I_{\tau}> \\
& \frac{\mathcal{M}_{D}^{2}}{m_{0}^{2}} \approx \frac{\sigma}{2 h_{0}}\left\{\left(\xi_{U}+h_{0} \xi_{D}\right) \rho-\left(\xi_{U}-h_{0} \xi_{D}\right) \frac{1}{\rho}\right\}+\xi_{1 / 2}<I_{\sigma}>
\end{aligned}
$$

with $\rho=\left(\frac{\tan \phi}{\tan \phi_{0}}\right)^{(1 / 5)}$. In the last two expressions we have used the convenient parametrisation $\mathcal{M}_{(U, D)_{0}}^{2}=\xi_{(U, D)} m_{0}^{2}$ and $m_{1 / 2}^{2}=\xi_{1 / 2} m_{0}^{2}$, while $\xi_{U} \equiv \xi_{H_{2}}+\xi_{Q}+\xi_{t^{c}}$ and $\xi_{D} \equiv \xi_{H_{1}}+\xi_{Q}+\xi_{b^{c}}$. Finally the integrals $<I_{\tau, \sigma}>$ are given by

$$
<I_{\tau}>=\frac{\tau}{2} \int_{t}^{t_{0}} \frac{G_{U}\left(t^{\prime}\right)}{\tau\left(t^{\prime}\right)}\left\{\left(1+\nu\left(t^{\prime}\right)\right) \frac{1}{\rho\left(t^{\prime}\right)}+\left(1-\nu\left(t^{\prime}\right)\right) \rho\left(t^{\prime}\right)\right\} d t^{\prime}
$$

and similarly for $\left\langle I_{\sigma}>\right.$ with the replacements $\tau \rightarrow \sigma$ and $G_{U} \rightarrow G_{D}, \nu \rightarrow \frac{1}{\nu}$ while $\nu$ is given by

$$
\nu=\left(\frac{\sqrt{1+u}-1}{\sqrt{1+u}+1}\right)^{\frac{1}{2}}
$$

The integrals $\left\langle I_{\tau, \sigma}>\right.$ look complicated, but in fact they can easily be performed using definitions (12,18) to express the functions $\rho, \nu, \tau, \sigma$ in terms of the integration variable $t^{\prime}$. As a matter of fact, for the case of interest $\left(h_{b} \sim h_{t}\right), u \gg 1$ the integrals can be simplified further, as $\nu \rightarrow 1$. Then, instead of $(\sqrt{72})$, we simply write

$$
<I_{\tau}>\approx \tau \int_{t}^{t_{0}} \frac{G_{U}\left(t^{\prime}\right)}{\tau\left(t^{\prime}\right) \rho\left(t^{\prime}\right)} d t^{\prime}, \quad<I_{\sigma}>\approx \sigma \int_{t}^{t_{0}} \frac{G_{D}\left(t^{\prime}\right)}{\sigma\left(t^{\prime}\right) \rho\left(t^{\prime}\right)} d t^{\prime}
$$


Once the solutions of the sums are obtained, we may substitute back into the equations for the individual masses. A simple way to solve these equations is achieved if we formally integrate the sums to obtain

$$
\begin{aligned}
& \mathcal{M}_{U}^{2}-\mathcal{M}_{U, 0}^{2}-C_{U}(t) m_{1 / 2}^{2}=-6 J_{U}-J_{D} \\
& \mathcal{M}_{D}^{2}-\mathcal{M}_{D, 0}^{2}-C_{D}(t) m_{1 / 2}^{2}=-J_{U}-6 J_{D}
\end{aligned}
$$

with $J_{I}(t)=\int h_{I}^{2} \mathcal{M}_{I}^{2} d t, I=U, D$. Now, the unknown integrals $J_{I}(t)$ can be expressed in terms of the already calculated sums, their initial conditions and known gauge functions, from the simple algebraic system (75,76). Then, the higgs mass parameters for example can be given from

$$
\begin{aligned}
& m_{H_{1}}^{2}=\left(\xi_{H_{1}}+C_{H}(t) \xi_{1 / 2}\right) m_{0}^{2}-3 J_{D}(t)+I_{S} \\
& m_{H_{2}}^{2}=\left(\xi_{H_{2}}+C_{H}(t) \xi_{1 / 2}\right) m_{0}^{2}-3 J_{U}(t)-I_{S}
\end{aligned}
$$

with $I_{S}$ representing the integral of the $S$-contribution in the case of non - universality. Similar expressions hold also for the other scalar masses of the third generation.

In order to examine the radiative breaking of the electroweak symmetry, we are finally interested for the mass parameters $\mu_{i}^{2}=m_{H_{i}}^{2}+\mu^{2}$. The initial value of $\mu$ is also unknown, but this arbitrariness can be eliminated by using the minimization conditions and determine its value in terms of known quantities. For the large $\beta$ we can write

$$
\mu^{2} \approx-m_{H_{2}}^{2}+\frac{m_{H_{1}}^{2}}{\tan ^{2} \beta}-\frac{1}{2} m_{Z}^{2}
$$

Notice that with the use of the relevant renormalization group equation for $\mu$ and (79), we may determine the initial condition for the $\mu$ parameter which may be useful in several cases.

Let us conclude this section with some remarks concerning the advantage of the above semi-analytic procedure with respect to the standard numerical integration. First we have shown that the useful quantities of the minimal susy model (Yukawa solutions, scalar masses, $\tan \beta, \mu$-term e.t.c.) can be expressed in terms only of known gauge functions and the parameter $u$. The value of the latter can be obtained easily at any scale in terms of the top and bottom couplings at the unification scale through the hypergeometric function defined in (14). In fact, in the limiting case we discuss in this work, the parameter $u$ can be expressed by a simple, approximately linear relation in terms of its initial value, thus a lot of information about the low energy parameters can be easily obtained by a straightforward calculation of the involved parameters. Furthermore, the scalar 
spectrum depends on two more new quantities, i.e. the integrals $I_{\tau, \sigma}$, which can also be easily computed or fitted by simple mathematical functions for any given $h_{(t, b)_{0}}$ set.

A final comment we wish to make here concerns the possible effects of the $A_{t, b}$ parameters in our solutions. We have already noted that, the $A_{t}-A_{b}$ renormalisation group equations can be solved independently in terms of the gauge and Yukawa couplings. In fact, the $A_{t, b}$ coupled differential system is exactly the same with that of the scalar sums (46) with the proper replacement of the coefficients $G_{U, D}$. After replacing their solutions $A_{t, b}=f_{t, b}\left(A_{t, 0}, A_{b, 0}\right)-I_{\tau, \sigma}^{\prime} m_{1 / 2}$, into the system of the scalar masses, one finds that the $\delta_{A_{i}}$ corrections are of the form (see also [26, 28])

$$
\delta_{A_{i}}=\delta_{i_{1}} A_{0}^{2}+\delta_{i_{2}} A_{0} m_{1 / 2}+\delta_{i_{3}} m_{1 / 2}^{2}
$$

The coefficients $\delta_{i_{(1,2,3)}}$ involve the two more integrals similar to $I_{\tau, \sigma}$ defined above. Remarkably, in the case of Yukawa couplings close to their infrared value, the coefficients multiplying the $A_{0}$ terms are found to be small $\delta_{i_{1,2}} \ll 1$. This result has also been confirmed elsewhere [26], where all terms involving the initial condition $A_{0}$ have been shown to be multiplied by a factor $1-\left(\frac{h_{t}}{h_{t}^{f x d}}\right)^{2}$, with $h_{t}^{f x d}$ the fixed point value of the top Yukawa coupling. For reasonable $A_{0}, m_{1 / 2}$ values, (i.e., not much larger than the gravitino mass), and staying close to the fixed point $h_{t}$-value, the above corrections do not modify substantially the present analysis. 


\section{Results and Conclusions}

We are ready now to present our numerical examples using the obtained scalar mass formulae. Although the formulae of the previous section look rather complicated, in fact for most of the cases of interest, they can be simplified considerably. As a matter of fact, the only "new" quantities that enter the various scalar masses are $J_{U, D}$ which mainly depend on the two integrals $I_{\tau}, I_{\sigma}$ and the initial boundary conditions $\xi_{i}$. For example, in the case of $h_{t} \sim h_{b}$ couplings close to their non-perturbative regime, the $J_{U, D}$ quantities can be easily simplified as follows

$$
\begin{aligned}
J_{U} & \approx \frac{1}{7}\left\{3.0+\epsilon_{\xi}+\left(C_{U}(t)-I_{\tau}\right) \xi_{1 / 2}\right\} m_{0}^{2} \\
J_{D} & \approx \frac{1}{7}\left\{3.0+\bar{\epsilon}_{\xi}+\left(C_{D}(t)-I_{\sigma}\right) \xi_{1 / 2}\right\} m_{0}^{2}
\end{aligned}
$$

where $\epsilon_{\xi}, \bar{\epsilon}_{\xi}$ represent small corrections of the order $\mathcal{O}(\sim 3 \%)$ which can be easily extracted from the previous formulae. Furthermore the $C_{U, D}=\sum_{n} C_{n}^{U, D}$ gauge functions are easily calculated from the formulae

$$
C_{n}(t)=\sum_{i=1}^{3} \frac{c_{i}^{n}}{2 b_{i} \alpha_{i_{G}}^{2}}\left(\alpha_{i}^{2}\left(t_{1}\right)-\alpha_{i}^{2}(t)\right), \quad n=1,2,3
$$

Thus $C_{U} \approx 7.1+6.7+0.5=14.3$ and similarly for $C_{D}$. Finally, for the region of interest the two integrals $I_{\tau, \sigma}$ are easily calculated. For $h_{t_{0}} \approx 3.0$ for example we find $I_{\tau} \approx 5.7$, while as $h_{b_{0}}<h_{t_{0}}$, we obtain $I_{\sigma}>I_{\tau}$. In the case of equal bottom-tau couplings the two expressions (81, 82) are almost identical (up to $\mathrm{U}(1)$ differences in $C_{U, D}$ gauge quantities and the $\epsilon_{\xi}, \bar{\epsilon}_{\xi}$ parameters which might differ due to non-universal initial boundary conditions). In the limiting case of equal b.c's at the GUT scale these quantities are equal and our expressions reduce to those of similar investigations of references [26, 6].

Let us now turn to the numerical investigation of the scalar masses. Using the the minimization conditions for the potential, it has been shown in section 2 that in order to achieve Radiative Symmetry Breaking at low energies, the constraint $\delta m_{H_{1,2}}^{2} \equiv m_{H_{1}}^{2}-m_{H_{2}}^{2} \geq m_{Z}^{2}$ should be satisfied. After some simple algebra we arrive at

$$
\begin{aligned}
\frac{\delta m_{H_{1,2}}^{2}}{m_{0}^{2}} & =\frac{1}{5}\left\{2\left(\xi_{H_{1}}-\xi_{H_{2}}\right)+3\left(\xi_{u^{c}}-\xi_{d^{c}}\right)\right\}+2 I_{S} \\
& -\frac{3}{10}\left[\left(\tau-\frac{\sigma}{h_{0}}\right) \rho\left(\xi_{U}+h_{0} \xi_{D}\right)+\left(\tau+\frac{\sigma}{h_{0}}\right) \frac{1}{\rho}\left(\xi_{U}-h_{0} \xi_{D}\right)\right] \\
& +\frac{3}{5}\left[\left(C_{u^{c}}-C_{d^{c}}\right)+I_{\sigma \tau}\right] \xi_{1 / 2}
\end{aligned}
$$


with the $I_{\sigma \tau}$ integral being the difference $I_{\sigma}-I_{\tau}$. In the above formulae, all functions depending on the parameter $u$ are finally expressed in terms of the initial value $u_{0}$, which depends only on the ratio of Yukawas, $h_{t, 0} / h_{b, 0}$ at $M_{G U T}$. Therefore, the constraint $m_{H_{1}}^{2}-m_{H_{2}}^{2}>m_{Z}^{2}$ has been reduced down to a simple algebraic inequality of the form

$$
\alpha \xi_{H_{1}}+\beta \xi_{H_{2}}+\gamma \xi_{1 / 2}+\delta>\xi_{Z}
$$

with $\xi_{Z}=\left(m_{Z} / m_{0}\right)^{2}$ while $\alpha, \beta, \gamma$ and $\delta$ can be easily read from (84). (The other constraints can also be easily converted to simple equations of this latter form). Notice that in the limit $h_{b} \rightarrow h_{t}$ the integrals $I_{\tau, \sigma}$ are approximately equal while $C_{u^{c}}, C_{d^{c}}$ differ only in the $U(1)$ factors. Therefore, in the case of non universality, in general we get $\gamma<\alpha, \beta$ from (85), and the main dependence is on $\xi_{H 1,2}$ parameters. For $h_{t} \geq h_{b}$ at $M_{U}$ we get $I_{\sigma \tau} \geq 0$, therefore the coefficient in front of $\xi_{1 / 2}$ is always positive. Furthermore, for universal boundary conditions $\xi_{H_{1}}=\xi_{H_{2}}, \xi_{u^{c}}=\xi_{d^{c}}$ the first line in the RHS of equation (84) becomes zero. In this case a lower bound is put on value of $\xi_{1 / 2}$ to satisfy (85). In any case, this condition can be naturally satisfied for non - universal boundary conditions and in particular if $\xi_{H_{1}}>\xi_{H_{2}}, \xi_{u^{c}} \geq \xi_{d^{c}}$. Thus, once a particular point $\left(\xi_{H_{1}}, \xi_{H_{2}}, \xi_{1 / 2}\right)$ is chosen, this condition may be seen as a lower bound on the scale $m_{3 / 2}$, i.e. $m_{3 / 2} \geq m_{Z} /\left(\alpha \xi_{H_{1}}+\beta \xi_{H_{2}}+\gamma \xi_{1 / 2}+\delta\right)^{1 / 2}$. In fig. 4 , this constraint is represented by a two dimensional surface as a function of the $\xi_{H_{1}}$ and $\xi_{1 / 2}$ parameters choosing two specific values for $\xi_{H_{2}}$. Evidently, for small $\xi_{1 / 2}$ the constraint is satisfied only for $\xi_{H_{1}}>\xi_{H_{2}}$.

We may further examine the Higgs mass term $m_{H_{2}}^{2}$ which should be negative in order to break the $S U(2)$ symmetry. If we substitute $J_{U}$ from the previous equations in (77), (ignoring for simplicity small corrections of the order $\mathcal{O}(2-$ $3 \%)$ ), we arrive at the result:

$$
\begin{aligned}
\frac{m_{H_{2}}^{2}}{m_{0}^{2}} & \approx \frac{1}{35}\left\{\left(17 \xi_{H_{2}}+3 \xi_{H_{1}}\right)-3\left(5 \xi_{Q}+6 \xi_{u^{c}}-\xi_{d^{c}}\right)\right\} \\
& -\frac{3}{7}\left\{C_{Q}+C_{u^{c}}-\frac{4}{3} C_{H}-I_{\tau}\right\} \xi_{1 / 2}
\end{aligned}
$$

After substituting the relevant functions by their numerical values, one can easily check under what conditions the required inequality $\left(m_{H_{2}}^{2}<0\right)$ is satisfied. For example assuming universal conditions for all scalars except $m_{H_{1}}$, a lower bound is put in the parameter $\xi_{1 / 2}$ only if $\xi_{H_{1}} \geq 4$ i.e., when $m_{H_{1}} \geq 2 m_{3 / 2}$.

Similarly, for the $\tilde{t}^{c}$ squark running mass we get

$$
\frac{\tilde{m}_{t^{c}}^{2}}{m_{0}^{2}} \approx \frac{1}{35}\left\{\left(23 \xi_{u^{c}}+2 \xi_{d^{c}}\right)-2\left(5 \xi_{Q}+6 \xi_{H_{2}}-\xi_{H_{1}}\right)\right\}
$$




$$
-\frac{2}{7}\left\{C_{Q}+C_{H}-\frac{5}{2} C_{u^{c}}-I_{\tau}\right\} \xi_{1 / 2}
$$

Now, if we denote with $\tilde{m}_{t^{c}}^{\text {exp. }}$ the lower experimental bound on the stop-mass, it is rather simple to investigate the constraint $\tilde{m}_{t^{c}}^{2}>\left(\tilde{m}_{t^{c}}^{2}\right)^{\text {exp. }}$ from the above formula. In fact, this can be also converted to a bound on the parameter $m_{3 / 2}$ as in the case of Eq.(85). In most of the parameter space however, this bound on $m_{0}^{2}$ is lower than the corresponding obtained from $\delta m_{H_{12}}^{2}$ condition. In fact, the $\left(\tilde{m}_{t^{c}}^{2}\right)^{\text {exp. }}$ - bound excludes only regions with $\xi_{1 / 2} \ll 1$.

Nevertheless, interesting bounds - in addition to $\xi_{H_{1}}>\xi_{H_{2}}$ - arise mainly from the $\tilde{m}_{b^{c}}-$ mass, given by

$$
\begin{aligned}
\frac{\tilde{m}_{b^{c}}^{2}}{m_{0}^{2}} & \approx \frac{1}{35}\left\{\left(23 \xi_{d^{c}}+2 \xi_{u^{c}}\right)-2\left(5 \xi_{Q}+6 \xi_{H_{1}}-\xi_{H_{2}}\right)\right\} \\
& -\frac{2}{7}\left\{C_{Q}+C_{H}-\frac{5}{2} C_{d^{c}}-I_{\sigma}\right\} \xi_{1 / 2}
\end{aligned}
$$

For many interesting regions of the $\xi_{i}$ parameter space the bounds obtained from this latter mass are non -trivial. Assume for example a moderate case where $\xi_{Q}=\xi_{d^{c}}=\xi_{H_{2}}=\frac{1}{2}$. (This would correspond to the relation $\tilde{m}_{i} \approx 0.7 m_{3 / 2}$ for the relevant mass parameters). Then the above formula implies the following relation between $\xi_{1 / 2}, \xi_{H_{1}}$

$$
\xi_{1 / 2} \geq \frac{2}{25} \xi_{H_{1}}+\frac{1}{30}\left[7\left(\frac{\tilde{m}_{b^{c}}^{e x p}}{m_{0}}\right)^{2}-\frac{17}{10}\right]
$$

where $\tilde{m}_{b^{c}}^{e x p}$ is the experimental lower bound of the sbottom mass. Now, if we imply a sensible value for $m_{H_{1}}$ (say $m_{H_{1}} \approx 2 m_{3 / 2}$ ) so as to satisfy naturally the condition(84), we should have at least $\xi_{1 / 2} \geq(0.3-0.4)$, no matter how small the ratio $\tilde{m}_{b^{c}}^{e x p} / m_{0}$ is. This in turn implies the constraint $m_{1 / 2} \geq(0.55-0.65) m_{3 / 2}$. Of course this holds for the particular set of $\xi_{i}$ 's chosen, but a detailed investigation can be easily done for the whole parameter space.

We should finally point out that the above results do not include contributions from a large $h_{\tau}$-Yukawa coupling which in various models is also predicted to be of the same order with $h_{t, b}$. A complete account of these effects in the described analytic procedure up to now was not possible. However, corrections of this Yukawa coupling are not expected to have a significant impact on the large part of the parameter space. Nevertheless, it will be a rather interesting question if the above analytic procedure can also be extended to include corrections of $h_{\tau}$, in the case where $h_{t} \sim h_{b} \sim h_{\tau}$.

In conclusion, in this paper we have presented analytic expressions for the Higgs mass parameters $m_{H_{1,2}}^{2}$ and squark masses of the third generation, when 
both top and bottom Yukawa couplings are large, as predicted by many unified theories. In the derivation of these expressions we have used exact analytic solutions of the top-bottom $\left(h_{t}, h_{b}\right)$ running Yukawa couplings ( expressed in terms of hypergeometric functions) which are involved in the evolution equations of the scalar masses. We have shown that, in the limit $h_{t}, h_{b} \gg h_{\tau}, \ldots$ the coupled differential system of Higgses and scalars quarks is reduced down to a simple first order Riccati Differential equation. Specific numerical examples have been presented in the interesting case of large $\tan \beta \approx \mathcal{O}(60)$, using values for the top coupling close to its non-perturbative regime. For these ranges of the parameters $h_{t}, \tan \beta$, and a bottom mass $m_{b} \approx 4.25 \mathrm{GeV}$, we predict a running top mass $m_{t} \approx(176-177) \mathrm{GeV}$ corresponding to a physical mass $m_{t}^{p h} \approx(184-185) \mathrm{GeV}$. These predictions can change substantially, if corrections from sparticle exchange loops (being proportional to $\tan \beta)$ are taken into account. They depend crucially on the value of the $\mu$ parameter, while they are mainly controlled by a linear combination of the $g_{3}^{2} m_{\tilde{g}}$ and $h_{t}^{2} A_{t}$ quantities, where $m_{\tilde{g}}, A_{t}$ are the gaugino mass and the trilinear coupling respectively. It is possible of course to assume proper boundary conditions to suppress those corrections down to a $(5-6) \%$ of the bottom mass, but in principle they can be as large as $40 \%$ is specific regions of the parameter space. A final answer to this question can be given only when the underlying theory is known to pick up a particular point of the parameter space. For the time being we can reverse the procedure and constrain the arbitrary parameters using the experimentally determined range of the top mass.

We have further presented simple expressions for the Higgses and scalars and discussed the constraints put by the radiative symmetry breaking mechanism, as well as, by charge and color protection on the initial conditions of the scalars. Thus the condition that the one Higgs (mass) ${ }^{2}$ turns negative at low energies can be easily satisfied for natural values of the scalar masses as can be concluded from (86). Furthermore, the second Higgs is protected from large negative corrections at low mass scales, either if we impose $m_{H_{1}}^{2}>m_{H_{2}}^{2}$ at the unification point, or if $m_{1 / 2}>m_{3 / 2}$. It is evident from (84) that the specific boundary conditions on the squark masses $\tilde{m}_{t^{c}}, \tilde{m}_{b^{c}}$, will play a role in the exact determination of the ranges of the above mass parameters. Finally $\tilde{m}_{Q}, \tilde{m}_{t^{c}}, \tilde{m}_{b^{c}}$ squark masses also impose additional constraints on the $\left(m_{3 / 2}, m_{1 / 2}\right)$ mass parameters. 


\section{References}

[1] H. P. Nilles, Phys. Rep. 110(1984)1;

H. E. Haber and G. L. Kane, Phys. Rep. 117(1985)75;

A. B. Lahanas and D. V. Nanopoulos, Phys. Rep. 145(1987)1;

S. Ferrara, ed., "Supersymmetry" (North-Holland, Amsterdam, 1987);

F. Zwirner, in "Proceedings of the 1991 Summer School in High Energy Physics and Cosmology", Trieste, 17 June, 9 August 1991 (E. Gava, K. Narain, S. Randjbar-Daemi, E. Sezgin and Q. Shafi, eds.), Vol. 1, p. 193.

[2] I. Antoniadis, J. Ellis, J. S. Hagelin and D.V. Nanopoulos Phys. Lett. B 231 (1989) 65; I. Antoniadis, et al, Phys.Lett. B 245 (1990) 161.

[3] G.K. Leontaris, J. Rizos and K. Tamvakis, Phys. Lett. B251(1990)83;

G.K. Leontaris and N.D. Tracas, Phys.Lett.B.260(1991)339;

A. E. Faraggi, Phys.Lett.B274 (1992) 47-52;

A. E. Faraggi, Top Quark Mass Prediction in Superstring Derived Standardlike Models, hep-ph/9506388.

[4] F. Abe et al, CDF coll., Phys. Rev. D 50 (1994) 2966; Phys.Rev.Lett. 73 (1994) 225; Fermilab-pub-95/022-E;

D0 coll., FERMILAB-PUB-95/028-E.

[5] B. Ananthanarayan, G. Lazarides and Q. Shafi, Phys. Rev.D44(1991)1613;

G. K. Leontaris and N. D. Tracas, Phys. Lett.B303(1993)50;

M. Carena, M. Olechowski, S. Pokorski and C. Wagner, Nucl. Phys. B 426 (1994) 269;

L. Hall, R. Rattazzi and U. Sarid, hep-ph/9405313;

M. Olechowski and S. Pokorski, Phys. Lett. B344(1995)201.

[6] C. Kounnas, I. Pavel, G. Ridolfi and F. Zwirner, Phys. Lett. B 354 (1995) 322.

[7] E. Cremmer, S. Ferrara, C. Kounnas and D.V. Nanopoulos, Phys. Lett. B133(1983)61.

J. Ellis, A.B. Lahanas, D. V. Nanopoulos and K. Tamvakis, Phys. Lett. B134 (1984) 429;

J. Ellis, C. Kounnas and D. V. Nanopoulos, Nucl. Phys. B241(1984)406 and B 247 (1984) 373. 
[8] C. Kounnas, I. Pavel and F. Zwirner, Phys. Lett. B 335 (1994) 403.

[9] G. K. Leontaris and N. D. Tracas, Phys. Lett. B351(1995)487.

[10] L. Ibáñez and G. G. Ross, Phys. Lett. B 110 (1982) 215;

K. Inoue et al, Prog. Theor. Phys. 68(1982)927;

L. Alvarez-Gaumé, M. Claudson and M.B. Wise, Nucl. Phys. B221(1983)495;

J. Ellis, J. S. Hagelin and K. Tamvakis, Phys. Lett. B125(1983)275;

L. Ibáñez and C. Lopez, Phys. Lett. B126(1983)54;

L. E. Ibáñez, Nucl. Phys. B218(1983) 514 ;

J. Ellis, J. S. Hagelin, D. V. Nanopoulos, and K. Tamvakis, Phys. Lett. B125 (1983) 275;

C. Kounnas, A. B. Lahanas, D. V. Nanopoulos, and M. Quirós, Phys. Lett. B132 (1983) 95; Nucl. Phys. B236(1984)438;

L. E. Ibáñez, C. Lopez and C. Muñoz, Nucl. Phys.B256 (1985) 218.

[11] L. E. Ibáñez and D. Lüst, Nucl. Phys. B382(1992)305;

A. Lleyda and C. Muñoz, Phys. Lett. B317(1993)82;

T. Kobayashi, D. Suematsu and Y. Yamagishi, Phys. Lett.B329(1994)27;

D. Matalliotakis and H. P. Nilles, Nucl. Phys. B435(1995)115;

M. Carena and C.E.M. Wagner, CERN preprint CERN-TH.7393/94;

[12] S. Dimopoulos and H. Georgi, Nucl. Phys. B193 (1981)150;

J. Ellis and D. V. Nanopoulos, Phys. Lett. B110(1982)44;

R. Barbieri and R. Gatto, Phys. Lett. B110(1982)211;

G. K. Leontaris, K. Tamvakis and J. D. Vergados, Phys. Lett. B 171 (1986) 412 ;

F. Gabbiani, and A. Masiero, Nucl.Phys.B322(1989)235;

T. S. Kosmas, G. K. Leontaris and J. D. Vergados, Progr. of Part. and Nucl. Phys. 33(1994)397-447;

D. Choudhury, F. Eberlein, A. Köning, J. Louis, and S. Pokorski, Phys. Lett. B342(1994)180

J. Louis and Y. Nir, preprint, hep-ph/9411429, LMU-TPW-94-17;

S. Dimopoulos and G.F. Giudice, hep-ph/9504296, CERN-TH/95-90.

[13] A.H. Chamseddine, R. Arnowitt and P. Nath, Phys. Rev. Lett. 49 (1982) 970;

R. Barbieri, S. Ferrara and C.A. Savoy, Phys. Lett. B119 (1982) 343; 
E. Cremmer, P. Fayet and L. Girardello, Phys. Lett. B122 (1983) 41;

L. Hall, J. Lykken and S. Weinberg, Phys. Rev. D27 (1983) 2359;

S.K. Soni and H.A. Weldon, Phys. Lett. B126 (1983) 215;

G.F. Giudice and A. Masiero, Phys. Lett. B206 (1988) 480.

[14] G. Gamberini, G. Ridolfi and F. Zwirner, Nucl. Phys. B331 (1990) 331.

[15] S. Ferrara, L. Girardello, C. Kounnas, and M.Porrati, Phys. Lett. B 192 (1987) 368;

I. Antoniadis, J. Ellis, E. G. Floratos, D. V. Nanopoulos and T. Tomaras, Phys. Lett.B191(1987)96;

S. Ferrara, C. Kounnas, and F. Zwirner, Nucl. Phys. B429(1994)589, and references therein.

[16] L. Ibáñez and C. Lopez, Nucl. Phys.B233(1984)511;

A. Bouquet, J. Kaplan and C. Savoy, B262(1985)299.

[17] G.K. Leontaris, Phys. Lett. B317(1993)569;

[18] E. G. Floratos and G.K. Leontaris, Phys. Lett. B336(1994)194;

[19] P. Binetruy and E. Dudas, Nucl. Phys. B442(1995)21.

[20] for recent numerical investigations see e.g.

R. Arnowit and P. Nath, Phys. Rev. Lett. 69 (1992) 725;

G. L. Kane, C. Kolda, L. Roszkowski and J. D. Wells, Phys. Rev. D49 (1994) 49;

V. Barger, M. S. Berger and P. Ohmann, Phys.Rev.D 49 (1994) 49084930;

D. J. Castano, E. J. Piard, and P. Ramond, Phys.Rev.D 49 (1994) 48824901.

[21] T. Banks, Nucl. Phys. B303(1988)172;

G. K. Leontaris, Phys. Lett. B236(1989)179.

[22] L. Hall, R. Rattazzi and U. Sarid, Phys. Rev. D50(1994)7048.

[23] M. Carena, M. Olechowski, S. Pokorski, and C. E. M. Wagner, Nucl.Phys.B419 (1994)213

[24] S. Martin and P. Ramond, Phys. Rev. D48(1993)5365. 
[25] I. Socolnikoff and R. Redheffer, Mathematics of Physics and Modern Engineering, McGraw-Hill, 1958.

[26] M. Carena, M. Olechowski, S. Pokorsky and C. Wagner, ref.[5]

G. K. Leontaris and N. D. Tracas, Phys. Lett.B342(1995)163;

M. Olechowski and S. Pokorski, ref.[5].

[27] I. Antoniadis, J. Ellis, J. S. Hagelin and D.V. Nanopoulos ref. [2]

[28] E. G. Floratos, G. K. Leontaris and S. Lola, hep-ph/9507402. 


\section{FIGURE CAPTIONS}

Figure 1. Plots of the ratio $r_{\beta}=\left(\tan \beta / \tan \beta_{0}\right)^{2 / 3}$ vs the parameter $u$ for selected ratios of the Yukawa couplings $h_{t, 0} / h_{b, 0}$.

Figure 2. The curve $A(u)=h(u)$ separating the two regions with $\dot{A}>0$ and $\dot{A}<0$.

Figure 3. Comparison of analytic approximate (upper curves) and numerical solutions (lower curves) of the ratios $\mathcal{X}(u) / m_{0}^{2}, \mathcal{Y}(u) / m_{0}^{2}$, for two cases of Yukawa couplings. (For $\mathcal{X}, \mathcal{Y}$ definitions see section 4 ).

Figure 4. Surfaces representing the lower $m_{0}^{2}$ bound, in the parameter space $\xi_{1 / 2}=m_{1 / 2}^{2} / m_{0}^{2}, \xi_{H_{1}}=m_{H_{1}}^{2} / m_{0}^{2}$ for $\xi_{H_{2}}=0.64$ (lower) and $\xi_{H_{2}}=1$ (upper case). If $\xi_{H_{2}} \geq \xi_{H_{1}}$, surface points exist only for $\xi_{1 / 2} \gg 1$. 
This figure "fig1-1.png" is available in "png" format from: http://arxiv.org/ps/hep-ph/9503455v2 


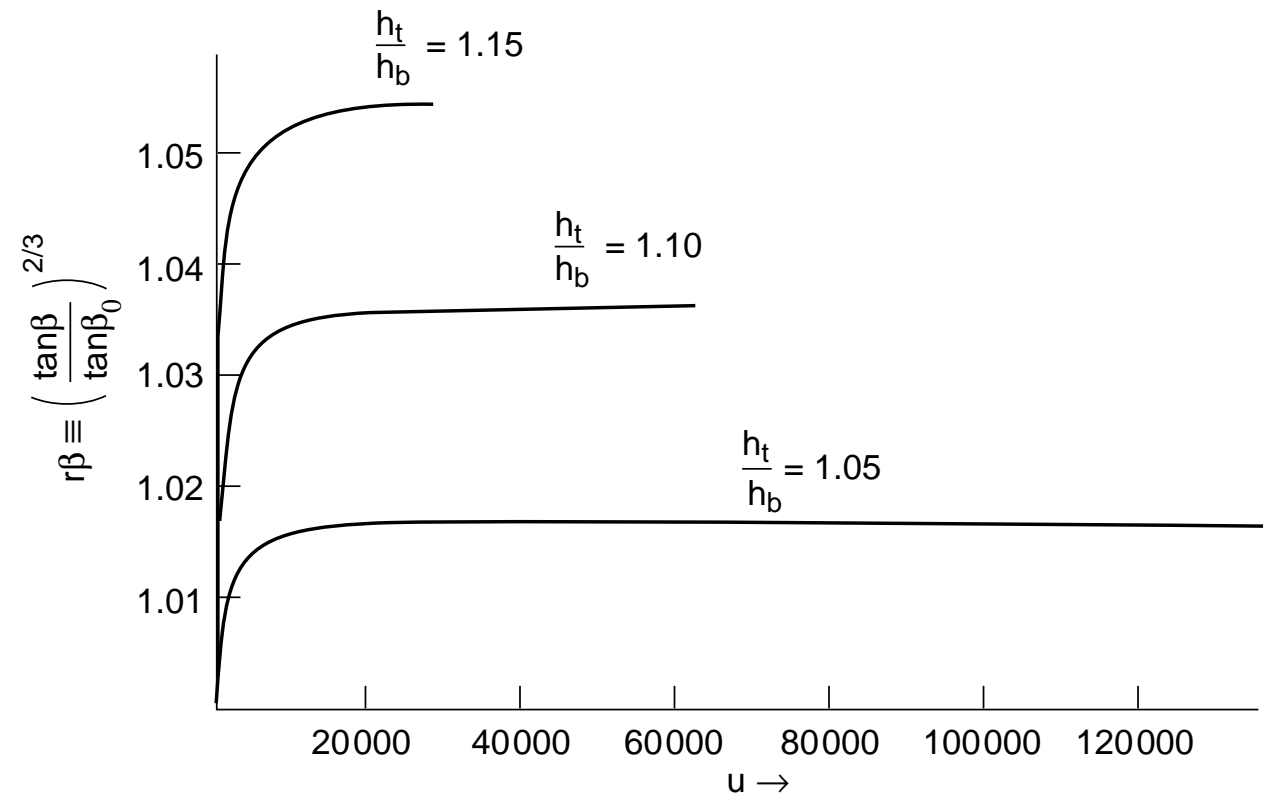

Fig. 1

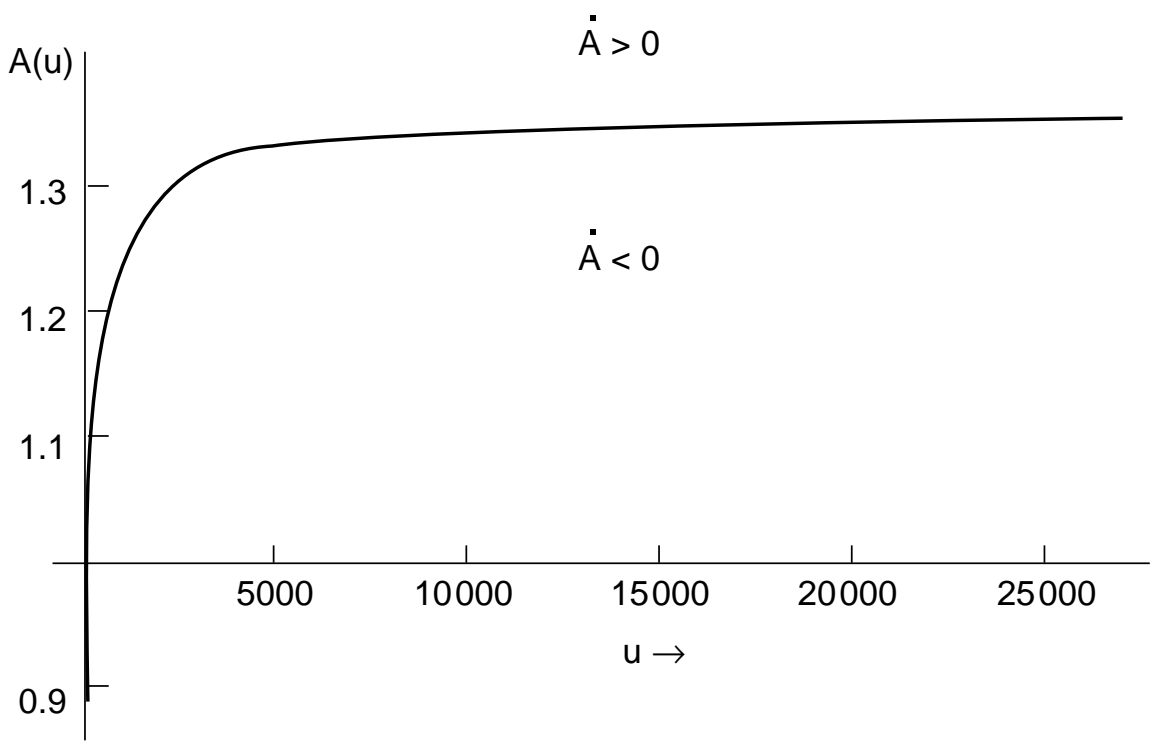

Fig. 2 
This figure "fig1-2.png" is available in "png" format from: http://arxiv.org/ps/hep-ph/9503455v2 

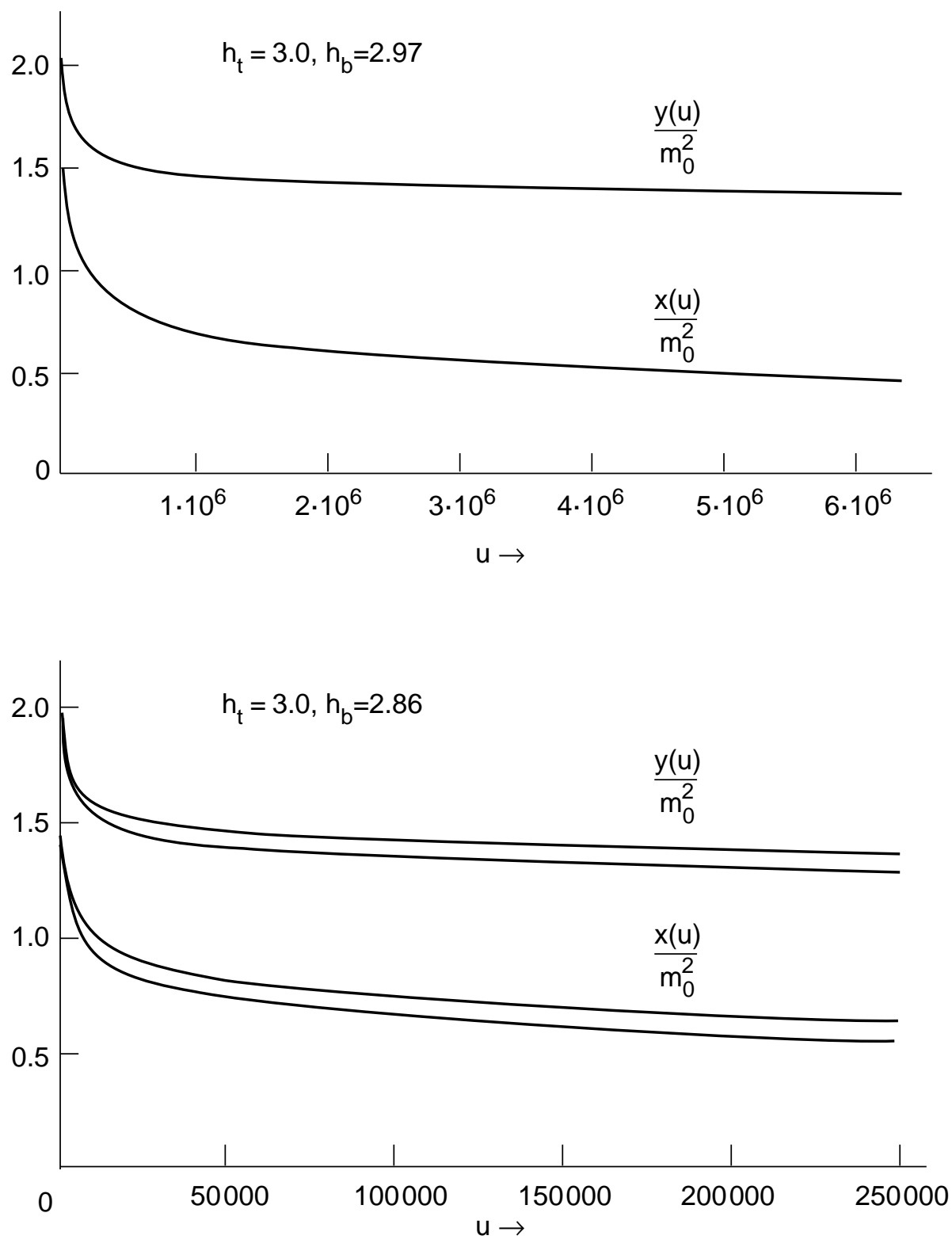

Fig. 3 
This figure "fig1-3.png" is available in "png" format from: http://arxiv.org/ps/hep-ph/9503455v2 

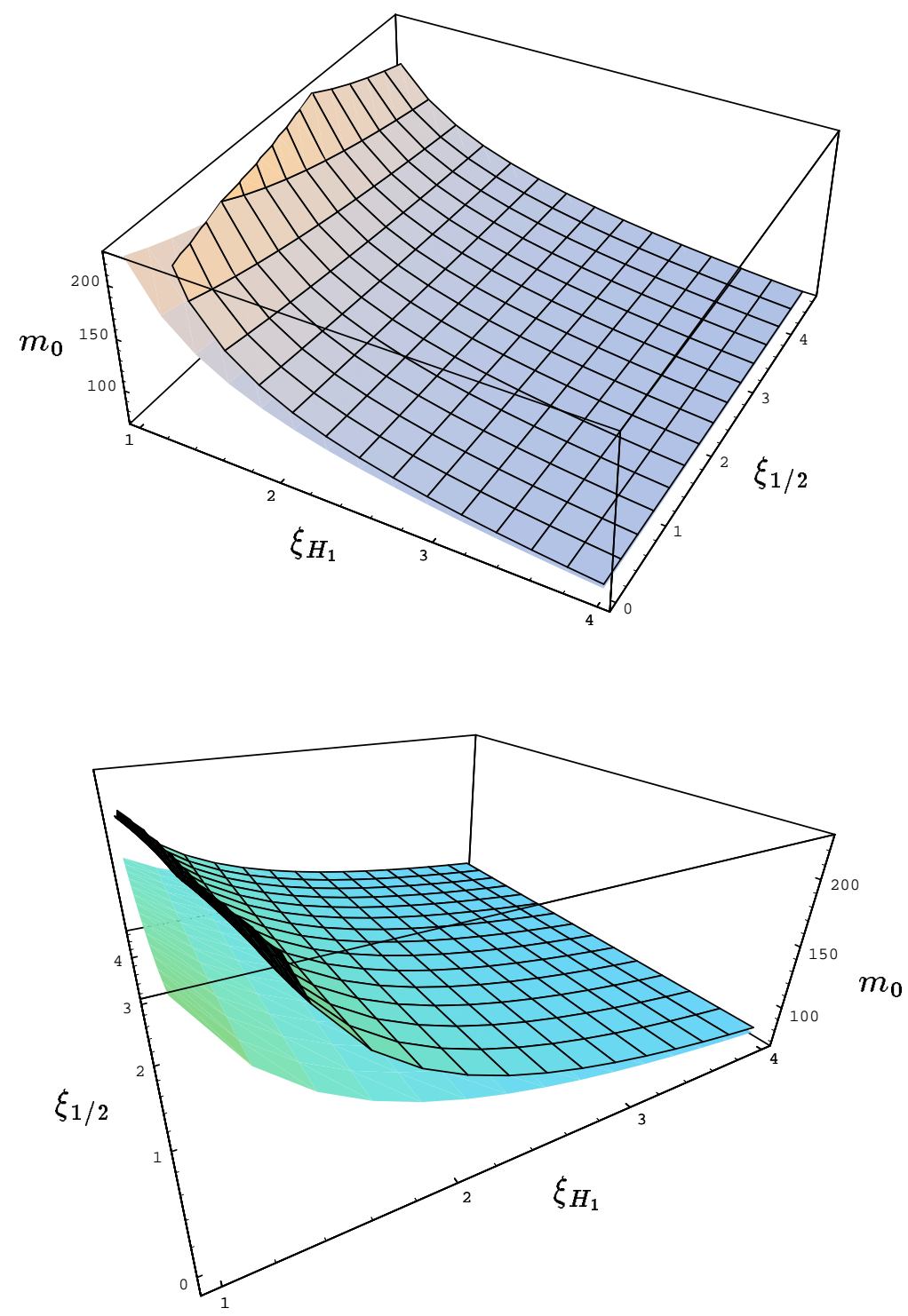

Figure 4 (from different viewpoints) 